\title{
Food and Water Consumption Result Standard Unit
}

National Cancer Institute

\section{Source}

National Cancer Institute. Food and Water Consumption Result Standard Unit. NCI

Thesaurus. Code C119841.

The standard unit of measure for food and water consumption results. 